\title{
PREDICTION OF LOW FREQUENCY BLOOD \\ PRESSURE OSCILLATIONS VIA A COMBINED HEART/RESISTANCE MODEL
}

\author{
John Ringwood ${ }^{1}$ Oliver Kinnane Simon Malpas ${ }^{* *}$ \\ Dept. of Electronic Eng., NUI Maynooth, Ireland \\ ** Dept. of Physiology, Uni. of Auckland, New Zealand
}

\begin{abstract}
Low frequency oscillations in blood pressure (BP) can occur due to a feedback pathway between the sensing of BP and the central nervous system (CNS), often termed the baroreflex, affecting both cardiac output (heart-rate and stroke volume) and peripheral resistance. In this paper, an integrated model of both these subsystems is assembled and an analysis technique developed, which shows the conditions under which a limit cycle oscillation can occur. In particular, the role of mean levels of cardiac output and peripheral resistance, previously thought to be relatively unimportant, in establishing and maintaining sustained oscillations, is highlighted. The ultimate aim of this analysis is to assist in the development of diagnostic tests based on measurement of low-frequency blood pressure oscillations. Copyright ${ }^{\circledR}$ 2005 IFAC
\end{abstract}

Keywords: Biomedical systems, limit cycles, oscillation, nonlinear analysis, blood pressure

\section{INTRODUCTION}

Over the last decade or so, it has been suggested that the measurement of low-frequency oscillations (circa $0.1 \mathrm{~Hz}$ in humans, sometimes known as Mayer waves) could help in the diagnosis of pathology or, at least, help in assessing the physiological state (Malliani et al., 1991). For example, it is known that the magnitude of oscillations increases in the case of haemorrhage (Malpas and Burgess, 2000) and the relatively straightforward measurement of blood pressure spectrum may reveal internal physiological state. For some time, researchers have striven to understand the origin of these oscillations and a variety of theories have been proposed, broadly separating into

1 The authors would like to acknowledge useful discussions with members of the Hamilton Institute at NUI Maynooth and also a Research Collaboration Grant provided by the Wellcome Trust philosophies which either suggest that the oscillation is produced centrally by the CNS (Cooley et al., 1998), or propose that a limit cycle exists around the neural blood-pressure control system (termed the baroreflex) (Kitney, 1979). It has been widely experimentally confirmed that the frequency of this low freq. oscillation decreases as species size increases and this has been seen to accord well with the limit cycle explanation (Ringwood and Malpas, 2001).

Both the heart and the peripheral resistance have the capability to facilitate neural control of blood pressure, via the 'Ohm's law' relationship:

$$
p_{b}(t)=q_{h}(t) r_{p}(t)
$$

where

$p_{b}(t)$ is mean arterial blood pressure,

$q_{h}(t)$ is cardiac output, and

$r_{p}(t)$ is total peripheral resistance of the circulatory system 


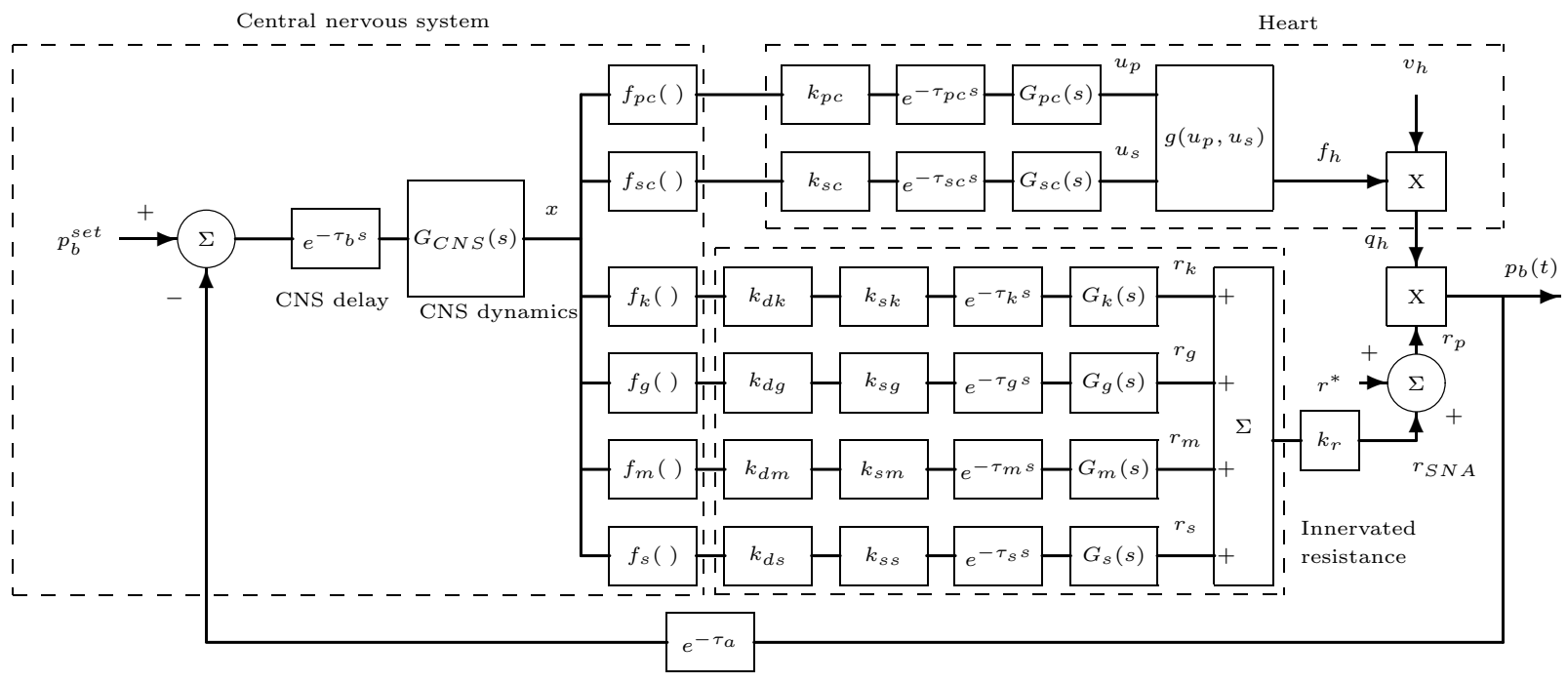

Fig. 1. Complete model of baroreflex, including heart and peripheral resistance paths

A variety of models, based on the limit cycle explanation and using various physiological components have been proposed. These include linear (Burgess et al., 1997) and nonlinear models (Ringwood and Malpas, 2001) based on the peripheral branch alone (Kitney, 1979) and models based on the cardiac side alone (Cavalcanti and Belardinelli, 1996). Some models also look at the combined cardiac/resistance system as a source of chaos (Seidel, 1997), while Liu et al. (2002) concludes that the cardiac side plays little role in the neural control of blood pressure around the frequencies of interest. Though the papers of Ringwood et al (Ringwood and Malpas, 2001) Burgess et al (Burgess et al., 1997) both develop analysis to show the conditions for oscillations, the former relies on a very specific relation between system (vasculature) and controller (neural) parameters, while the latter has a milder set of conditions based on a describing function analysis.

This paper essentially extends the model reported in (Ringwood and Malpas, 2001) in two ways:

- The complete peripheral resistance system is modelled, rather then just the renal vasculature, and

- The cardiac side is included, comprising both heart-rate and stroke volume influences.

Though a simple describing function analysis is no longer possible, due to the more complicated structure of the enlarged system, an analysis technique is developed to examine the conditions for limit cycle development. This is the focus of the paper and allows important conclusions to be drawn regarding the relative influences (and interactions) of the cardiac and resistance branches.
In particular, the important role of mean cardiac output and mean peripheral resistance, previously thought to be relatively unimportant, is revealed.

The paper is laid out as follows: An integrated model for cardiac output and total peripheral resistance is developed in Section 2, while the 'describing function-like' analysis is developed in Section 3, with a numerical example used to confirm its accuracy. Finally, Section 4 articulates the impact of the results from the analysis and makes final conclusions.

\section{A COMPLETE BAROREFLEX MODEL}

A model for the complete neural baroreflex, which has been assembled from experimentally validated sub-components, is shown in Fig.1. The model is parameterised for the rabbit, due to the best availability of parameter values for this species. In broad terms, the model can be divided into components representing the heart, the vasculature (peripheral resistance) and the central nervous system. Note that the peripheral resistance divides up into components which respond to sympathetic nerve activity (SNA) [the innervated resistance, $\left.r_{S N A}\right]$ and that which is independent of SNA, $r^{*}$. Innvervated resistance accounts for approximately $70 \%$ of total peripheral resistance and is distributed among the various beds (Sherwood, 1997) as shown in Table 1. In addition, the various beds have different sensitivities (Ninomiya et al., 1971), also documented in Table 1 . Note that the dc gains of the dynamical transfer functions, $G_{k}, G_{g}, G_{m}$ and $G_{s}$, are all normalised to unity. The low-pass dynamics of these transfer 
Table 1. Innervated resistance beds

\begin{tabular}{ccccc}
\hline Bed & Kidney & Gut & Muscle & Skin \\
\hline Identifier & $k_{d k}$ & $k_{d g}$ & $k_{d m}$ & $k_{d s}$ \\
Distribution & 0.2 & 0.27 & 0.15 & 0.09 \\
\hline \hline Identifier & $k_{s k}$ & $k_{s g}$ & $k_{s m}$ & $k_{s s}$ \\
Sensitivity & 0.9 & 0.25 & 0.9 & 0.1 \\
\hline
\end{tabular}

functions are specified (Guild et al., 2001; Stauss et al., 1999) as:

$$
G_{m}(s)=\frac{11 s^{2}+6.64 s+1}{4.27 s^{4}+21 s^{3}+36 s^{2}+22 s+1}
$$

with $G_{k}(s)=G_{g}(s)=G_{m}(s)$, and

$$
G_{s}(s)=\frac{1}{1.87 s+1}
$$

The delays in the various beds, including those corresponding to parasympathetic and sympathetic action to the heart and the afferent delay (in the feedback path) are enumerated (Liu et al., 2002; Ikeda et al., 1996) in Table 2. Note that

Table 2. Delay values

\begin{tabular}{cccccc}
\hline Identifier & $\tau_{a}$ & $\tau_{b}$ & $\tau_{p c}$ & $\tau_{s c}$ & $\tau_{k}, \tau_{g}, \tau_{m}, \tau_{s}$ \\
\hline Value & 0.2 & 0.4 & 0.3 & 0.8 & 0.85 \\
\hline
\end{tabular}

the value used for the vascular beds represents an average for the distributed innervated subsystems (e.g. skin). The baroreflex curves, realised as sigmoidal curves of the form:

$$
f(x)=\frac{l}{1+e^{-\beta x}}-\frac{l}{1+e^{\beta x}}+f^{o}
$$

are parameterised (Head and McCarty, 1987) as in Table 3 , with curvature expressed by $\beta$, vertical range by $l$ and vertical offset by $f^{o}$. The remain-

Table 3. Baroreflex curve parameters

\begin{tabular}{ccc}
\hline Parasymp. & Car. Symp. & Resistance Symp. \\
\hline$\beta_{p c}=-0.14$ & $\beta_{s c}=0.04$ & $\beta_{k}=\beta_{g}=\beta_{m}=\beta_{s}=0.04$ \\
$l_{p c}=65.5$ & $l_{s c}=42.5$ & $l_{k}=l_{g}=l_{m}=l_{s}=42.5$ \\
$f_{p c}^{o}=65.5$ & $f_{s c}^{o}=42.5$ & $f_{k}^{o}=f_{g}^{o}=f_{m}^{o}=f_{s}^{o}=102$ \\
\hline
\end{tabular}

ing two components of the peripheral resistance subsystem are the mean peripheral resistance not under neural control, $r^{*}=0.12 \mathrm{mmHg} \mathrm{min} / \mathrm{ml}$, and the scaling gain, $k_{r}=0.0012$, used to transpose SNA values to peripheral resistance units.

On the cardiac side, the remaining dynamic components (Ikeda et al., 1996; Kawada et al., 1996) are:

$$
\begin{aligned}
G_{C N S}(s) & =\frac{1.33 s+1}{s+1} \\
G_{p c}(s) & =\frac{1}{1.22 s+1} \\
G_{s c}(s) & =\frac{1}{1.29 s^{2}+1.92 s+1}
\end{aligned}
$$

while the scaling gains used to transform PSNA and SNA values to nerve stimulation values are:

$$
k_{p c}=0.148, k_{s c}=0.181
$$

Finally, stroke volume, $v_{h}$, is assumed constant (Suga et al., 1976) at $2.5 \mathrm{ml} /$ beat and the sympatho-vagal relationship, $g\left(u_{p}, u_{s}\right)$, was determined by fitting a nonlinear curve (Levy, 1971; Seidel, 1997) of the form:

$$
\begin{array}{r}
f_{h}=\alpha_{1} \tanh \left(\gamma_{1} u_{s}\right)\left(1-\tanh \left(\gamma_{2} u_{p}\right)\right)- \\
\alpha_{2} \tanh \left(\gamma_{3} u_{p}\right)+f_{h}^{o}
\end{array}
$$

to the data recorded by Kawada et al. (1999) for the rabbit, giving the coefficients in Table 4 . $f_{h}^{o}$ has a value of 245.8 , representing the (mean) autorhythmic heart rate of the rabbit.

Table 4. Sympatho-vagal parameters

\begin{tabular}{cccccc}
\hline Parameter & $\alpha_{1}$ & $\alpha_{2}$ & $\gamma_{1}$ & $\gamma_{2}$ & $\gamma_{3}$ \\
\hline Value & 181 & 156.1 & 0.04 & 0.035 & 0.07 \\
\hline
\end{tabular}

\section{A CONDITION FOR LF OSCILLATION}

The model of Fig.1 has common components in some of its branches and, in general, is too cumbersome to examine in terms of limit cycles, so the simplified model of Fig. 2 is proposed, which still retains the individuality of cardiac (sympathetic and parasympathetic) and peripheral resistance branches, but is more tractable with a view to analysis. It will be shown that this approximation introduces negligible inaccuracy with respect to prediction of oscillations. In this simplified representation, $G_{r}(s)$ is as given in (2), while $k_{d r}=$ 0.39 , determined as the sum of the products of the distribution and sensitivity gains for the individual innervated resistance paths. Furthermore, $g\left(u_{p}, u_{s}\right)$ is linearised to the affine function:

$$
g^{*}\left(u_{p}, u_{s}\right)=k_{s} u_{s}-k_{p} u_{p}+f_{h}^{o}
$$

with $k_{p}=-1.2$ and $k_{s}=0.88$, since it contains no inflections and is therefore unlikely to contribute to a limit cycle (it is also not significantly nonlinear).

A conventional describing function (DF) approach, which can be used to reveal conditions for limit cycle oscillations, cannot be directly applied to the system of Fig.2 due to the multiple paths with individual dynamics and nonlinear characteristics. Some modest extension of the DF method is possible beyond the classic single loop analysis (Atherton, 1982), but the baroreflex system presents a more significant challenge. The approach adopted is to assume a sinusoidal input at point $x$, evaluate the feedback return from this 


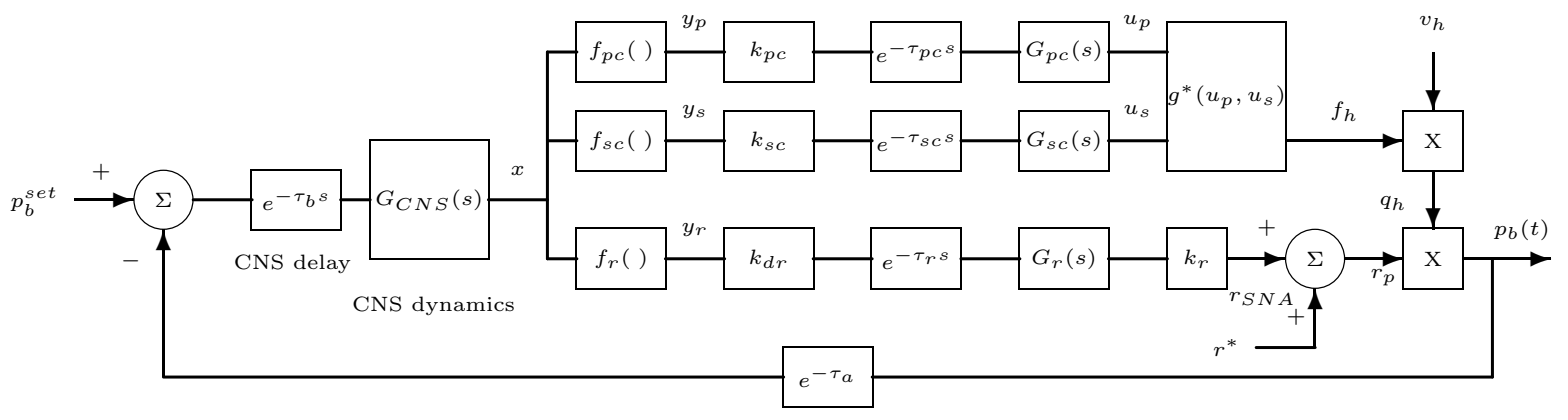

Fig. 2. Simplified model of baroreflex, including heart and peripheral resistance paths

excitation and equate it to the original signal, therefore solving for limit cycle conditions. It will be assumed (in the spirit of the DF) that (a) harmonics arising in the nonlinearities described by $f_{p c}(), f_{s c}()$ and $f_{r}()$ and (b) sum frequencies arising in the multiplier at the system output have negligible influence over a possible limit cycle, due to the low pass nature of $G_{p c}, G_{s c}$ and $G_{r}$. This is confirmed from examination of the spectrum of $x(t)(X(\omega))$ during a typical limit cycle, as shown in Fig.3. Assume the input to the nonlinearities is

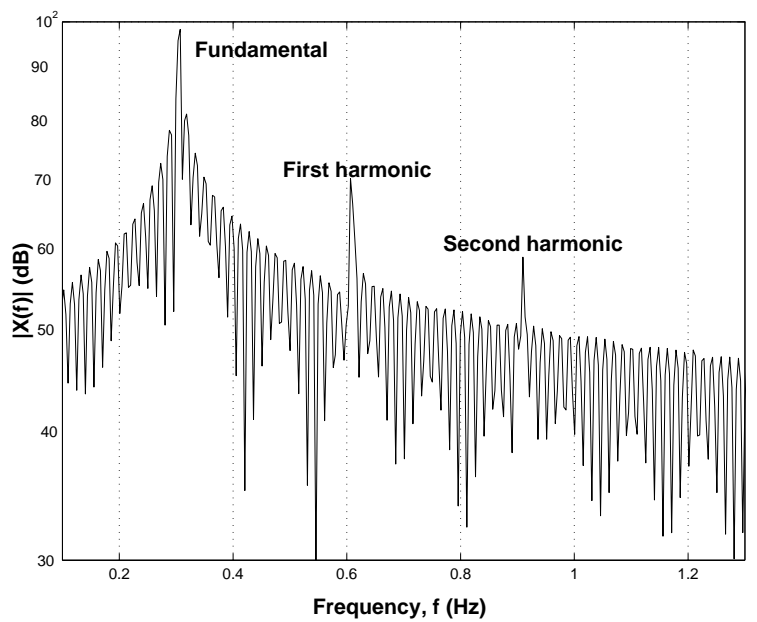

Fig. 3. Spectrum of $x(t)$ during a limit cycle

sinusoidal:

$$
x(t)=A \sin [\omega t]
$$

Then, in the spirit of the DF, the output of each nonlinearity is given by:

$$
\begin{aligned}
& y_{p}(t)=N_{p}(A) A \sin [\omega t]+f_{p c}^{o} \\
& y_{s}(t)=N_{s}(A) A \sin [\omega t]+f_{s c}^{o} \\
& y_{r}(t)=N_{r}(A) A \sin [\omega t]+f_{r}^{o}
\end{aligned}
$$

Note that since $f_{p c}(), f_{s c}()$ and $f_{r}()$ are all odd, the describing functions $N_{p}, N_{s}$ and $N_{r}$ are all real and independent of the input frequency, $\omega$ (Slotine and Li, 1990). Now,

$$
f_{h}(t)=\overline{f_{h}}+
$$

$$
\begin{aligned}
& k_{s}\left|G_{s c}(\omega)\right| k_{s c} N_{s}(A) A \sin \left[\omega t+\angle G_{s c}(\omega)-\omega \tau_{s c}\right]- \\
& \quad k_{p}\left|G_{p c}(\omega)\right| k_{p c} N_{p}(A) A \sin \left[\omega t+\angle G_{p c}(\omega)-\omega \tau_{p c}\right](15)
\end{aligned}
$$

where

$$
\overline{f_{h}}=f_{h}^{o}+k_{s} k_{s c} f_{s c}^{o}-k k_{p} k_{p c} f_{p c}^{o}
$$

Equation (15) can be represented, with the obvious identification of terms, as:

$$
\begin{aligned}
f_{h}(t) & =K_{s} \sin \left[\omega t+\phi_{s c}\right]-K_{p} \sin \left[\omega t+\phi_{p c}\right]+\overline{f_{h}} \\
& =K_{c} \sin \left[\omega t+\phi_{c}\right]+\overline{f_{h}}
\end{aligned}
$$

where

$$
\begin{aligned}
K_{c} & =\sqrt{K_{s}^{2}+K_{p}^{2}-2 K_{s} K_{p} \cos \left[\phi_{s c}-\phi_{p c}\right]} \\
\phi_{c} & =\tan ^{-1} \frac{K_{s} \cos \left[\phi_{s c}\right]-K_{p} \cos \left[\phi_{p c}\right]}{K_{p} \sin \left[\phi_{p c}\right]-K_{s} \sin \left[\phi_{s c}\right]}
\end{aligned}
$$

Similarly,

$r_{p}(t)=k_{r}\left|G_{r}(\omega)\right| k_{d r} N_{r}(A) \sin \left[\omega t+\angle G_{r}(\omega)-\omega \tau_{r}\right]+\bar{r}(20)$

where

$$
\bar{r}=r^{*}+k_{r} k_{d r} f_{r}^{o}
$$

or

$$
r_{p}(t)=K_{r} \sin \left[\omega t+\phi_{r}\right]+\bar{r}
$$

Using (17) and (22) gives:

$$
\begin{aligned}
p_{b}(t) & =v_{h} \bar{r} \overline{f_{h}} \\
& +v_{h} K_{r} K_{c} \sin \left[\omega t+\phi_{r}\right] \sin \left[\omega t+\phi_{c}\right] \\
& +v_{h} \overline{f_{h}} K_{r} \sin \left[\omega t+\phi_{r}\right]+v_{h} \bar{r} K_{c} \sin \left[\omega t+\phi_{c}\right](23)
\end{aligned}
$$

Ignoring the double frequency term at $2 \omega$ gives:

$$
\begin{aligned}
p_{b}(t) & =v_{h} \bar{r} \overline{f_{h}}+v_{h} K_{r} K_{c} \cos \left[\phi_{r}-\phi_{c}\right] \\
& +v_{h} \overline{f_{h}} K_{r} \sin \left[\omega t+\phi_{r}\right]+v_{h} \bar{r} K_{c} \sin \left[\omega t+\phi_{c}\right]
\end{aligned}
$$

or, with accumulation of terms:

$$
\begin{aligned}
p_{b}(t) & =v_{h} \bar{r} \overline{f_{h}}+\kappa(\omega) \\
& +K_{r}^{*} \sin \left[\omega t+\phi_{r}\right]+K_{c}^{*} \sin \left[\omega t+\phi_{c}\right]
\end{aligned}
$$

or

$$
p_{b}(t)=v_{h} \bar{r} \overline{f_{h}}+\kappa(\omega)+K_{p}^{*} \sin \left[\omega t+\phi_{p}\right]
$$


with

$$
\begin{aligned}
K_{p}^{*} & =\sqrt{K_{r}^{* 2}+K_{c}^{* 2}+2 K_{c}^{*} K_{r}^{*} \cos \left[\phi_{c}-\phi_{r}\right]}(27) \\
\phi_{p} & =\tan ^{-1} \frac{K_{r}^{*} \cos \left[\phi_{r}\right]+K_{c}^{*} \cos \left[\phi_{c}\right]}{-\left(K_{r}^{*} \sin \left[\phi_{r}\right]+K_{c}^{*} \sin \left[\phi_{c}\right]\right)}
\end{aligned}
$$

Continuing around the feedback path, we finally arrive at:

$$
\begin{aligned}
& x_{r e t}(t)=\left|G_{C N S}(\omega)\right|\left\{\left(p_{b}^{\text {set }}-v_{h} \bar{r} \overline{f_{h}}-\kappa(\omega)\right)-\right. \\
& \left.K_{p}^{*} \sin \left[\omega t+\phi_{p}-\omega\left(\tau_{a}+\tau_{b}\right)+\angle G_{C N S}(\omega)\right]\right\}
\end{aligned}
$$

The requirement for sustained oscillation is:

$$
x_{\text {ret }}(t)=x(t)
$$

which, from (11) and (29) gives the three conditions:

$$
\begin{aligned}
\left|G_{C N S}(\omega)\right|\left(p_{b}^{s e t}-v_{h} \bar{r} \overline{f_{h}}-\kappa(\omega)\right) & =0 \\
\left|G_{C N S}(\omega)\right| K_{p}^{*} & =A \\
\phi_{p}-\omega\left(\tau_{a}+\tau_{b}\right)+\angle G_{C N S}(\omega)+\pi & =0
\end{aligned}
$$

Some comments on these equations are appropriate:

- Equation (31) merely establishes a d.c. equilibrium around the system of Fig.3, subject to a frequency dependent term, $\kappa(\omega)$.

- Equations (31) to (33) are a set of nonlinear coupled equations in both $\omega$ and $A$. Though (32) appears to just contain magnitude terms, $K_{p}^{*}$ contains phase terms, as evident in (27). Similarly, magnitude terms (which depend on $A$ via the describing function terms) are included in (33) via $\phi_{p}$.

- The presence of $\pi$ (due to negative feedback) is interesting, since it can be offset by an arbitrary addition (or subtraction) of $\pi$ from $\phi_{p}$ in (28), since $\tan [\gamma]=\tan [\gamma+\pi]$. This could correspond to positive feedback !

Ideally, we would like to solve (31) to (33) analytically for $\omega$ and $A$, to determine relationships between the physiological parameters of the system and the presence/absence and/or magnitude and frequency of blood pressure oscillations. However, this presents some difficulties, since:

- Equations are nonlinear and may not admit a unique solution,

- The equations represent extremely complex relationships between the model parameters and the unknowns, $\omega$ and $A$, via equations (15) to (28), and

- The complexity of the equations is further exacerbated by the need for an analytical approximation for the describing function terms in (12) to (14).

The describing function problem is significant, since no exact closed-form analytical expression exists for the describing function of a sigmoidtype nonlinearity. In an attempt to preserve the analytical relationship between model parameters and oscillation characteristics (for a peripheral resistance only model), various sigmoid approximations have been developed (Kinnane et al., 2004), but all involve series expansions, which are unwieldy. However, the intricate mix of terms involving both cardiac and peripheral resistance components shows the collective importance of both sides in sustaining oscillations.

A simulation of the nominal system, with a blood pressure setpoint, $p_{b}^{\text {set }}$, of $80 \mathrm{mmHg}$, as in Fig.1 reveals a $0.34 \mathrm{~Hz}$ oscillation in blood pressure, with amplitude $9 \mathrm{mmHg}$ (typical of experimental evidence (Malpas and Burgess, 2000)). This is confirmed by equations (31) to (33) as follows:

$$
\begin{aligned}
1.26(80-(2.5)(0.16)(202)+0.033) & =-1.08(\mathbf{0}) \\
(1.26)(9.1) & =11.46(\mathbf{1 1 . 6}) \\
-1.353-2.14(0.2+0.4)+0.1+\pi & =0.6(\mathbf{0})
\end{aligned}
$$

with the close equality (correct values in bold) confirming the validity of the adoption of the reduced model of Fig.2 for the purpose of stability analysis. The Fourier integrals in the DFs of (12) to (14) were evaluated using the trapezoidal rule.

The cases where either cardiac or peripheral resistance side were inhibited were also examined, with a disappearance of the oscillation in both cases. This was the case even when the mean nerve 'tone' (due to $f_{p c}^{o}, f_{s c}^{o}, f_{k}^{o}, f_{g}^{o}, f_{m}^{o}$ and $f_{s}^{o}$ ) were retained, indicating the clear inter-dependence of both sides in sustaining oscillations.

\section{CONCLUSIONS}

This paper has given some insight into the production of low frequency oscillations in blood pressure via a limit cycle by assembling a comprehensive model containing all relevant subsystems and developing an analysis tool to examine for the presence/absence of oscillations. The model itself gives significant insight into the mechanisms and components which may be important in the generation of limit cycles. For example, the model clearly shows that mean levels, via the multiplication blocks (see Fig.1), play an important role in mediating oscillations, as well as gain. It is also clear, both from the model and from simulation studies on the model, that the cardiac side has an important role to play in mediating oscillations, contrary to some previous conclusions. Indeed, the conclusion (Liu et al., 2002) that the cardiac side has little influence, based on the fact that a vagotomy and suppression of cardiac SNA resulted in little change in the frequency response relating renal SNA to blood pressure, is easily explained 
by our model, with an alternative conclusion. In the experiments of Liu et al. (2002), mean heart rate was found to drop from 265 bpm to 206 , which gives an effective mean reduction in cardiac output of $3 \mathrm{~dB}$, which is almost exactly the mean gain reduction in renal frequency response (51 $\rightarrow 48.8 \mathrm{~dB}$ ). However, this gives little information about the ability of the cardiac loop to sustain oscillations itself. Simulation studies, in fact, confirm that oscillation (under nominal conditions) is primarily due to the gain in the cardiac branch.

Finally, the mathematical conditions for oscillation in (31) to (33) are a little disappointing in that clear analytical relationships between the physiological parameters and oscillation characteristics are difficult to deduce. However, numerical results confirm the validity of the reduced model in Fig.2 for development of the criterion and future work will focus on the numerical solution of these coupled nonlinear equations.

\section{REFERENCES}

Atherton, D.P. (1982). Nonlinear Control Engineering. Van Nostrand Reinhold.

Burgess, D.E., J.D. Hundley, S.-G. Li, D.C. Randall and D.R. Brown (1997). First-order differential-delay equation for the baroreflex predicts the $0.4 \mathrm{hz}$ blood pressure rhythm in rats. Am. J. Physiol. 273, R1878-R1884.

Cavalcanti, S. and E. Belardinelli (1996). Modelling of cardiovascular variability using a differential delay equation. IEEE Trans. on Biomed. Eng. 43, 982-989.

Cooley, R.L., N. Montano, C. Cogliati, P. Van de Borne, W. Richenbacher, R. Oren and V.K. Somers (1998). Evidence for the central origin of the low-frequency oscillation in rrinterval variability. Circulation 96, 556-561.

Guild, S.-J., P.C. Austin, M. Navakatikyan, J.V. Ringwood and S.C. Malpas (2001). Dynamic relationship between sympathetic nerve activity and renal blood flow: a frequency domain approach. Am. J. Physiol. 281, R206R212.

Head, G. and R. McCarty (1987). Vagal and sympathetic components of the heart rate range and gain of the baroreceptor-heart rate reflex in conscious rats. J. Auton. Nerv. Syst. 21, 203-213.

Ikeda, Y., T. Kawada, T. Sugimachi, O. Kawaguchi, T. Shishido, T. Sato, H. Miyano, W. Matsuura, J. Alexander and K. Sunagawa (1996). Nueral arc of baroreflex optimises dynamic pressure regulation in achieving both stability and quickness. $A m$. J. Physiol. 40, H882-H890.

Kawada, T., M. Sugimachi, T. Shishido, H. Miyano, T. Sato,
R. Yoshimura, H. Miyashita, T. Nakahara, J. Alexander and K. Sunagawa (1999). Simultaneous identification of static and dynamic vagosympathetic interactions in regulating heart rate. Am. J. Physiol. 276, R782R789.

Kawada, T., Y. Ikeda, M. Sugimachi, T. Shishido, O. Kawaguchi, T. Yamazaki, J. Alexander and K. Sunagawa (1996). Differential dynamic baroreflex regulation of cardiac and renal sympathetic nerve activities. Am. J. Physiol. 40, H288-H295.

Kinnane, O., J.V. Ringwood, D. Kelly and S. Malpas (2004). Describing function approximation for biomedical engineering applications. In: Proc. Irish Signals and Systems Conf.. pp. 125-247. IEE. Belfast.

Kitney, R.I. (1979). A nonlinear model for studying oscillations in the blood pressure control system. J. Biomed. Eng. 1, 89-99.

Levy, M.N. (1971). Sympathetic-parasympathetic interactions in the heart. Circ. Res. 29, 437445.

Liu, H.-K., S.-J. Guild, J.V. Ringwood, C.J. Barrett, B.L. Leonard, S.-K. Nguang, M.A. Navakatikyan and S.C. Malpas (2002). Dynamic baroreflex control of blood pressure: influence of the heart vs. peripheral resistance. Am. J. Physiol. 283, R533-R542.

Malliani, A., M. Pagani, F. Lombardi and S. Cerutti (1991). Cardiovascular neural regulation explored in the frequency domain. Circulation 84, 482-492.

Malpas, S.C. and D.E. Burgess (2000). Renal sna as the primary mediator of slow oscillations in blood pressure during hemorrhage. Am. J. Physiol. 279, H1299-H1306.

Ninomiya, I., N. Nisimaru and H. Irisawa (1971). Sympathetic nerve activity to spleen, kidney and heart in response to baroreceptor input. Am. J. Physiol. 221, 1346-1351.

Ringwood, J.V. and S.C. Malpas (2001). Control of renal blood flow - the case for a nonlinear model. Am. J. Physiol. 280, R1105-R1115.

Seidel, H. (1997). Nonlinear dynamics of physiological rhythms. Lagos Verlag.

Sherwood, L. (1997). Human Physiology. 3rd ed.. Wadsworth.

Slotine, J.-J. and W. Li (1990). Applied Nonlinear Control. Pearson.

Stauss, H.M., J.U. Stegmann, P.B. Persson and H.J. Habler (1999). Frequency response characteristics of sympathetic transmission to skin vascular smooth muscle in rats. Am. J. Physiol. 277, R591-R600.

Suga, H., H. Sagawa and A. Shoukas (1976). Carotid sinus baroreflex effects on instantaneous pressure-volume ratio of the canine left ventricle. J. Physiol. Soc. Japan 65, 106-107. 